\author{
A Thesis \\ entitled \\ Lifetimes and Oscillator Strengths for Ultraviolet Transitions \\ in Singly Ionized Copper \\ by \\ Michael S. Brown
}

As partial fulfillment of the requirements for the
Master of Science in Physics

Advisor: Dr. Steven R. Federman

College of Graduate Studies

The University of Toledo

May 2009 

An Abstract of

\title{
Lifetimes and Oscillator Strengths for Ultraviolet Transitions in Singly Ionized Copper
}

\author{
Michael S. Brown \\ Submitted in partial fulfillment \\ of the requirements for the \\ Master of Science in Physics \\ The University of Toledo \\ May 2009
}

Information on the makeup of the solid material in space as well as on the production of elements in our Galaxy and beyond may be gained through analysis of ultraviolet absorption from ions in interstellar clouds. High quality oscillator strengths are needed to convert the amount of absorption into an accurate abundance from data acquired with the Hubble Space Telescope and the Far Ultraviolet Spectroscopic Explorer. Lifetimes from my beam-foil experiments for ultraviolet transitions in $\mathrm{Cu}$ II are reported. The Cu II results for the $1358.773 \AA$ line $\left(3 \mathrm{~d}^{10}{ }^{1} \mathrm{~S}_{0}-3 \mathrm{~d}^{9} 4 \mathrm{p}{ }^{1} \mathrm{P}_{1}^{\mathrm{o}}\right)$ provide further evidence for a short lifetime for the upper level of interest. Additional results for transitions that can repopulate the upper state of interest indicate the data are not affected by these cascades. My measured lifetime of $1.37 \pm 0.04 \mathrm{~ns}$ is in excellent agreement with the most recent experimental results and is consistent with theoretical values. My determination of $0.273 \pm 0.008$ for the oscillator strength is in very good agreement with the recommended value given by Morton (2003) and the theoretical results of Donnelly et al. (1999). 


\section{Acknowledgments}

There are many people I would like to thank for all of their support during the course of this project. My thanks to my advisor, Dr. Steve Federman, for his perseverance in keeping me on track and his patience in not giving up on me. Thanks to Drs. Rick Irving and Song Cheng for all their help keeping the accelerator and all its components up and running. Many thanks to Dr. Scott Lee for agreeing to sit on my committee and most especially for all the advice and help he has provided to me over my many years here, both as an undergraduate and graduate student. Thanks to all the faculty and staff members of the Department of Physics and Astronomy, who have all helped in one way or another. Finally, thanks to my family and friends

for all the encouragement they have given me over the many years it has taken to finish this thesis. 


\section{Contents}

Abstract

Acknowledgments $\quad$ iii

Contents $\quad$ iv

List of Figures $\quad$ vi

List of Tables $\quad$ vii

1 Introductory Material 1

2 Experimental Setup $\quad 3$

3 Data Acquisition and Analysis $\quad 9$

3.1 Primary Line of Interest . . . . . . . . . . . . . . . . 9

3.2 Cascade Repopulation . . . . . . . . . . . . . . . . . . . . 12

4 Results and Discussion $\quad 16$

4.1 Comparison of Lifetimes and Branching Ratios . . . . . . . . . . . . 16

4.2 Comparison of Oscillator Strengths . . . . . . . . . . . . . . . 18 
4.3 Concluding Remarks . . . . . . . . . . . . . . . . . . . 20

References 


\section{List of Figures}

2-1 A schematic of the general layout of The University of Toledo Heavy Ion Accelerator, THIA (Haar, et al. 1993) . . . . . . . . . . . . 4

3-1 Spectra of Cu II line at $1358 \AA$ with Gaussian fit. . . . . . . . . . . . 10

3-2 Decay curve with two exponential fit of $\mathrm{Cu}$ II line at $1358 \AA$ at 170 $\mathrm{keV}$ in the forward direction. . . . . . . . . . . . . . . . . 11

3-3 Decay curve with two exponential fit of $\mathrm{Cu}$ II line at $1358 \AA$ at 170 keV in the reverse direction. . . . . . . . . . . . . . . . . . . . . 12

3-4 Decay curve with two exponential fit of $\mathrm{Cu}$ II line at $1358 \AA$ at 240 $\mathrm{keV}$ in the forward direction. . . . . . . . . . . . . . . . 13

3-5 Semi-log plot with fit of forward decay curve at $170 \mathrm{keV}$ for $\lambda 2048$. $\quad 14$

3-6 Semi-log plot with fit of forward decay curve at $170 \mathrm{keV}$ for $\lambda 2255$. $\quad 14$ 


\section{List of Tables}

2.1 Grating Specifications . . . . . . . . . . . . . . 5

2.2 Detector Specifications . . . . . . . . . . . . . . 5

4.1 Measured and Calculated Lifetimes and Branching Fractions of Cu II 18

4.2 Oscillator Strengths for the $3 \mathrm{~d}^{10}{ }^{1} \mathrm{~S}_{0}-3 \mathrm{~d}^{9} 4 \mathrm{p}{ }^{1} \mathrm{P}_{1}^{\mathrm{o}}$ transition of $\mathrm{Cu}$ II . 


\section{Chapter 1}

\section{Introductory Material}

Characterizing the interstellar medium (ISM) can give us information on the makeup of interstellar clouds, the abundance of elements and the production of elements in our Galaxy. One way of determining this information is the analysis of ultraviolet absorption from ions in interstellar clouds and the general ISM. The goal of this thesis has been to make accurate lifetime measurements and accurate determinations of the oscillator strength for the $3 \mathrm{~d}^{10}{ }^{1} \mathrm{~S}_{0}-3 \mathrm{~d}^{9} 4 \mathrm{p}{ }^{1} \mathrm{P}_{1}^{\mathrm{o}}$ transition in $\mathrm{Cu}$ II at $1358.773 \AA$. With an oscillator strength precisely determined, an accurate value for the gas phase abundance of $\mathrm{Cu}$ in the ISM can be determined. The percentage of the element that is incorporated into grains is then inferred through comparison of the observed gas phase abundance in all ionization stages to the solar abundance (Spizter, 1978).

Previous studies of the interstellar abundance of copper (Jenkins et al. 1986; Car-

tledge et al. 2006) relied on atomic oscillator strengths compiled by Morton and colleagues (Morton \& Smith 1973; Morton 1991; Morton 2003). For the line of inter- 
est, there is a significant spread in experimental (Curtis et al. 1976; Kono \& Hattori 1982; Pinnington et al. 1997) and theoretical (Theodosiou 1986; Loginov 1993; Pinnington et al. 1997; Donnelly et al. 1999; Biémont et al. 2000; Dong \& Fritzsche 2005) lifetimes and the associated oscillator strengths. In order to help clarify the situation, I conducted a new set of measurements.

One must make a precise measurement of the lifetime of the excited state of interest to obtain a value for the oscillator strength (or $f$-value). The technique I use to measure lifetimes of excited states is beam-foil spectroscopy (BFS). I discuss the details of BFS in the next chapter. Once the lifetime is known, the $f$-value can be derived.

In the following chapters, the details of the experiment are discussed. In Chapter 2 the experimental setup and technique used to acquire data is described, while in Chapter 3 the data analysis techniques and the details of how to infer the uncertainty in the experiment are presented. In Chapter 4, comparisons of my measured lifetime and oscillator strength to previously reported measurements and calculations are given, followed by a brief discussion of the results and their implications for the determination of the abundance of $\mathrm{Cu}$ in the ISM. 


\section{Chapter 2}

\section{Experimental Setup}

The Toledo Heavy Ion Accelerator (THIA) is used to generate the ions for the experiments. Figure 2-1 shows an overview of the accelerator and its associated hardware. The accelerator is a Danfysik Model 1080 mass separator and ion accelerator with a Danfysik Model 911A ion source attached. The 911A ion source is a hollow cathode design that is capable of generating ions from gaseous, liquid or solid source materials. The mass separating magnet allows for an isotopically pure beam to be passed from the ion source to the acceleration column without contamination from unwanted ion species. Energy is imparted to the ions by putting a high voltage on the accelerator terminal. This is accomplished by using a high voltage power supply (Glassman Model PG300P001-LR) that can output up to $300 \mathrm{kV}$ at up to $1 \mathrm{~mA}$.

Once the ions are accelerated with the desired voltage, they are steered into the foil chamber. The foil chamber contains a carriage that is precisely moveable parallel and anti-parallel to the path of the ion beam. The carriage has an attached rotating wheel, which can hold up to 23 foil holders that can be rotated, one at a time, into 


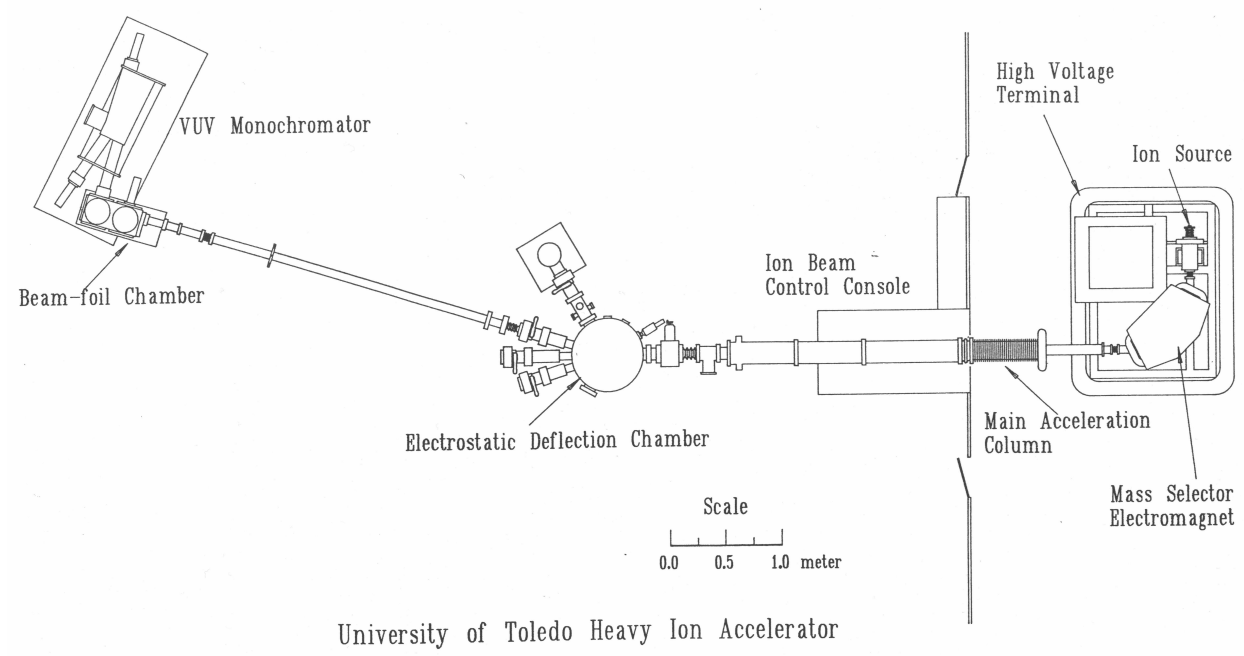

Figure 2-1: A schematic of the general layout of The University of Toledo Heavy Ion Accelerator, THIA (Haar, et al. 1993)

the path of the ion beam and perpendicular to it. Just after the foil holders is the optical monitor (OM), a fiber optic bundle mounted just below the path of the beam. The signal from this fiber optic bundle is connected to an ultraviolet sensitive (solar blind) photo-multiplier tube (PMT). The OM signal is used to normalize the data against the total light output. The Faraday cup (FC) is fixed to the back of the foil chamber and can be used as a second means of data normalization. Another function of the $\mathrm{FC}$ is to stop the ions from impacting the back of the chamber.

Attached to the side of the foil chamber is the entrance slit to a $1 \mathrm{~m}, \mathrm{f} / 10.4$ normal incidence, Acton vacuum monochromator (model VM-521-SG). Light enters the monochromator through a narrow entrance slit and is dispersed by a grating. Three different gratings are available for use in this monochromator system. The 
characteristics of the three different gratings are summarized in Table 2.1.

Table 2.1: Grating Specifications

\begin{tabular}{|c|c|c|}
\hline Groves $/ \mathbf{m m}$ & Wavelength Region $(\AA)$ & Max Resolution $(\AA)$ \\
\hline 2400 & $450-1600$ & 0.07 \\
1200 & $1600-3200$ & 0.14 \\
600 & $3200-6500$ & 0.28 \\
\hline
\end{tabular}

The dispersed light then passes through the exit slits and is collected by a detector. There are three different detectors available for data acquisition. Table 2.2 contains a summary of the characteristics of these detectors.

Table 2.2: Detector Specifications

\begin{tabular}{|l|c|}
\hline Detector & Wavelength Region $(\AA)$ \\
\hline Galileo Channeltron & $450-2000$ \\
Hammamatsu R7154 PMT & $1600-3200$ \\
Bialkali PMT & $3200-6500$ \\
\hline
\end{tabular}

The signals from the detector are then passed through a preamplifier, an amplifier and a discriminitor to data collection electronics. Then the signals pass through a Jorway Model 73A Camac Crate controller and are finally stored and displayed on a data acquisition computer.

The data acquisition computer uses Labview to control the movement of the foil wheel carriage and the rotation of the grating during the experiments. There are two types of measurements that can be taken with this system: spectral measurements and lifetime measurements. Spectra are taken to determine the wavelength at which an ion is radiating light. Once the wavelength region of interest has been determined, the monochromator is set up with the appropriate grating and detector for that 
wavelength region. A broad scan is taken over the region to see if any lines can be detected and to determine if there is any offset between the wavelength dial reading and the actual wavelength being detected. Once the line or lines of interest are detected, and the offset is taken into account, multiple scans of the region are acquired. As discussed earlier, the OM is used to determine whether or not a particular data set is acceptable to be included in the analysis. If the fluctuations in the OM of a given data set are within $\pm 20 \%$ of the average value of all data points in the OM, the data set is considered to be acceptable. Each acceptable scan is normalized and all the normalized scans are then summed. This continues until enough data are acquired to make an accurate determination of the line center for the peak of interest. The method of determining the line center is described in the next chapter. Once the line center is determined, the grating is set to that wavelength so that lifetime measurements can be taken. For the most part, the limiting factor for the width of the region being measured is the finite life of the foils.

Lifetime measurements are made by fixing the grating to disperse a particular wavelength and by moving the foil carriage in discrete steps with respect to the entrance slit. This allows snapshots of the light to be obtained at various distances downstream of the foil. Since the distance downstream is known and the post-foil velocity of the beam can be calculated, the post-foil time can be determined by dividing the distance in $\mathrm{mm}$ by the velocity in $\mathrm{mm} / \mathrm{ns}$ to give the time for any data point. The typical maximum distance from the foil that is measured in these experiments is about $25 \mathrm{~mm}$ set by the size of the chamber. Another limitation is that the FC is fixed with respect to the foil. As the foil moves away from the FC, 
the post-foil beam dispersion results in less ions being collected by the FC and an apparent decrease in the beam current. A significant change in the current over the course of a scan will typically result in that scan being unusable for data analysis.

In order to account for systematic errors, three different types of lifetime measurements are taken: forward lifetime measurements, reverse lifetime measurements at the same energy, and forward lifetime measurements at a significantly different energy. The forward lifetime measurements consist of moving the foil from a position slightly downstream of the monochromator entrance slit to a position upstream of the slit. How far upstream depends on the lifetime being measured. Longer lifetimes mean the foil needs to be moved further upstream. The reverse lifetime measurement consists of moving the foil from upstream of the slit to a position just downstream of the slit (i.e., the opposite of the forward lifetime measurement technique.) Two reasons for doing this are to account for any distortion in the time scale due to foil thickening and to check for the effects of foil aging (Federman et al. 1992). The third measurement consists of repeating the forward lifetime measurement at a different energy. Lifetimes measured at two different energies allows us to check for the effects of foil aging, for effects due to different energy losses in the foil, and for the effects of different amounts of beam divergence (Federman et al. 1992).

As stated above, the lifetime of each foil is a limiting factor in the measurements that have been made. The estimated foil life can be calculated using the program Beaming2. By inputting the atomic number, atomic mass and the acceleration energy of the incident ion as well as the foil material and foil thickness, the program outputs the post-foil values for the energy, velocity, estimated foil life and the percentages of 
the post foil charge states of the ions. Using this information, the estimated foil life is used to determine how long a particular scan should last, how many data points should be scanned, what size steps should be taken for each measurement and at what energy to run. The foils used for these experiments are made from thin carbon films deposited on microscope slides. The foils have an areal density that varies from slide to slide but are in the range of $2.1 \mu \mathrm{g} / \mathrm{cm}^{2}$ to $2.5 \mu \mathrm{g} / \mathrm{cm}^{2}$. When trying to decide the parameters of a data scan, how long the foil lasts is a large concern. Scanning a wider wavelength region or measuring a longer lifetime may require larger steps to be taken between data points, shorter dwell times to be used for each data point or both. 


\section{Chapter 3}

\section{Data Acquisition and Analysis}

\subsection{Primary Line of Interest}

For this investigation, I started by scanning in the region of the $1358.773 \AA$ line of $\mathrm{Cu}$ II. I detected a peak in the appropriate wavelength region and acquired several scans. These scans were normalized to try to correct for any fluctuations in the ion beam or any change in the characteristics of the foil that could affect the beam, as noted in the previous chapter. The scans were then summed and fitted using the normal distribution function (Bevington \& Robinson 1992)

$$
I(\lambda)=\frac{1}{\sigma \sqrt{2 \pi}} e^{\frac{-\left(\lambda-\lambda_{0}\right)^{2}}{2 \sigma^{2}}}
$$

where $I(\lambda)$ is the intensity at any given wavelength, $\lambda$ is the value of the data point in $\AA, \lambda_{0}$ is the line center in $\AA$, and $\sigma$ is the Gaussian width of the feature. The summed scan with fit is shown in Figure 3-1. 


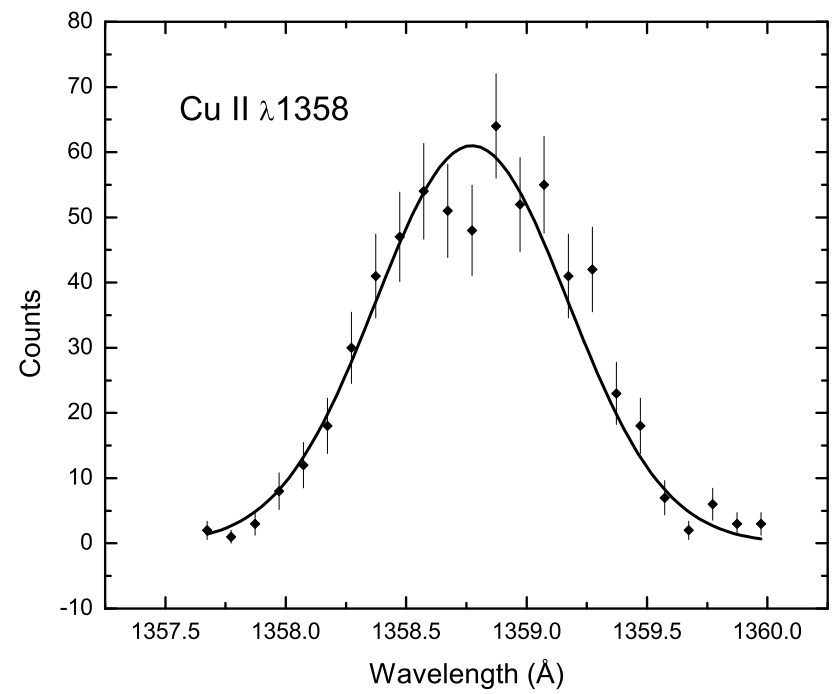

Figure 3-1: Spectra of Cu II line at $1358 \AA$ with Gaussian fit.

Once the line of interest was found, the 2400 line/mm grating was positioned at what was determined to be the peak of the spectra. I then began to acquire measurements of the lifetime of the excited state for this transition with the channeltron detector. For this phase of the investigation, the ions were accelerated with $170 \mathrm{keV}$ of energy and scanned in the forward direction. I normalized the data and summed 43 of the scans with a total acquisition time of 281 seconds. I started the analysis using a single exponential fit to the data and found the result did not fit the data well. A good fit is one in which the reduced chi squared, $\frac{\chi^{2}}{\nu}$, is equal to or close to one. The quantity $\nu$ is the number of degrees of freedom, which depends on the number of data points and the number of fitted parameters. The equation to solve for a single exponential (Bevington \& Robinson 1992) is

$$
N(t)=N_{0} e^{\frac{-t}{\tau}}
$$




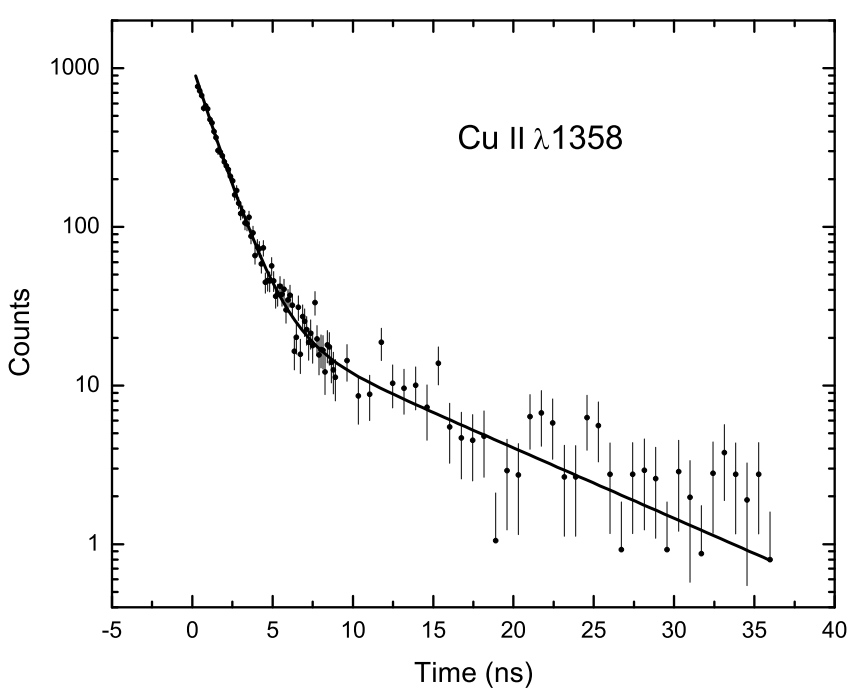

Figure 3-2: Decay curve with two exponential fit of $\mathrm{Cu}$ II line at $1358 \AA$ at $170 \mathrm{keV}$ in the forward direction.

where $N(t)$ is the number of counts at any given time, $N_{0}$ is the maximum number of counts, $t$ is the time in ns, and $\tau$ is the mean lifetime in ns. I then fit the data using a two exponential fit. The fit resulted in a reduced $\chi^{2}$ value of 1.07 which indicated a good fit. Next I fit the data using a three exponential fit and found no improvement in the fit and therefore concluded that a two exponential decay function gave the best fit. The resulting lifetimes were determined to be $1.36 \pm 0.04 \mathrm{~ns}$ for the primary decay of interest and $9.82 \pm 0.94$ ns for the secondary decay. The decay curve fitted with two exponentials can be seen in Figure 3-2.

In order to check for systematic errors, I acquired more data using the same energy but with the foil wheel carriage moving in the reverse direction. I normalized and summed 18 scans with a total acquisition time of 87 seconds and fit the curve. Using the same procedure as before, I again concluded a two exponential decay function 


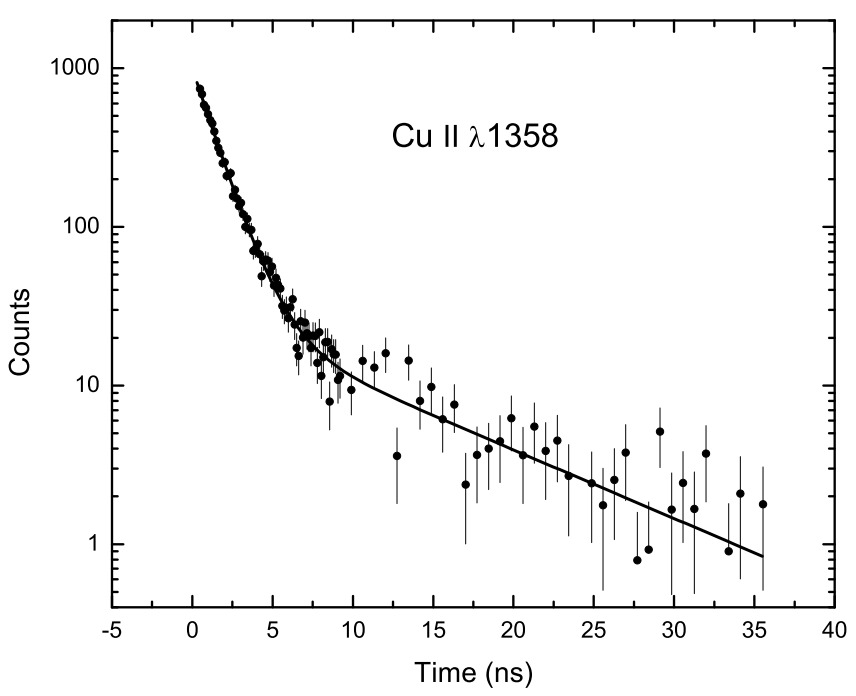

Figure 3-3: Decay curve with two exponential fit of $\mathrm{Cu}$ II line at $1358 \AA$ at $170 \mathrm{keV}$ in the reverse direction.

gave the best fit with lifetimes of $1.38 \pm 0.04 \mathrm{~ns}$ for the primary and $9.95 \pm 0.99 \mathrm{~ns}$ for the secondary. The summed curve and fit are shown in Figure 3-3. For the third part of the investigation, I again scanned in the forward direction, but now the energy was increased to $240 \mathrm{keV}$. I normalized and summed 6 scans with a total acquisition time of 28 seconds. I used the same procedure for analysis as before and again found that a two exponential decay gave the best fit. The results give lifetimes of $1.36 \pm$ $0.05 \mathrm{~ns}$ for the primary and $9.49 \pm 1.18 \mathrm{~ns}$ for the secondary. The summed curve and fit are shown in Figure 3-4.

\subsection{Cascade Repopulation}

The next phase of the investigation involved looking at possible contributions to the population in the upper level of interest $\left(4 \mathrm{p}{ }^{1} \mathrm{P}_{1}^{\mathrm{o}}\right)$ from cascades involving higher 


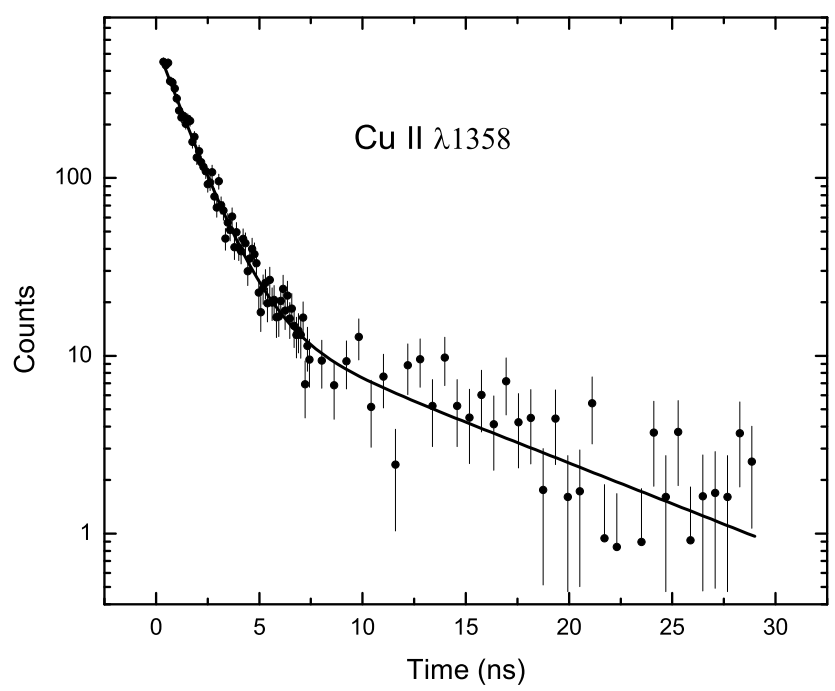

Figure 3-4: Decay curve with two exponential fit of $\mathrm{Cu}$ II line at $1358 \AA$ at $240 \mathrm{keV}$ in the forward direction.

energy levels. The longer lived decay could arise from a cascading transition. Three possible transitions that could contribute to the upper level of primary interest were identified. The wavelengths were located at approximately $2048 \AA$ (4d $\left.{ }^{1} \mathrm{~S}_{0}\right), 2225$ $\AA\left(4 \mathrm{~d}{ }^{3} \mathrm{~F}_{2}\right)$, and $2255 \AA\left(4 \mathrm{~d}^{3} \mathrm{D}_{1}\right)$. Using the same procedures as before, I scanned each of the three regions using the solar blind PMT and was able to acquire data for the lines at 2048 and $2255 \AA$. The line at $2225 \AA$ was blended with another line and was not resolvable. I background subtracted, normalized and summed the data for the 2048 and $2255 \AA$ lines, and found lifetimes of $1.88 \pm 0.06 \mathrm{~ns}$ and $1.65 \pm 0.13$ ns, respectively. These data were obtained in the forward direction at $170 \mathrm{keV}$; for $\lambda 2048$, 7 scans totaling $15.1 \mathrm{~s}$ were used, and for $\lambda 2255$, there were 7 scans totaling 19 s. The summed curves with fits for these two lines are shown in Figures 3-5 and 3-6. 


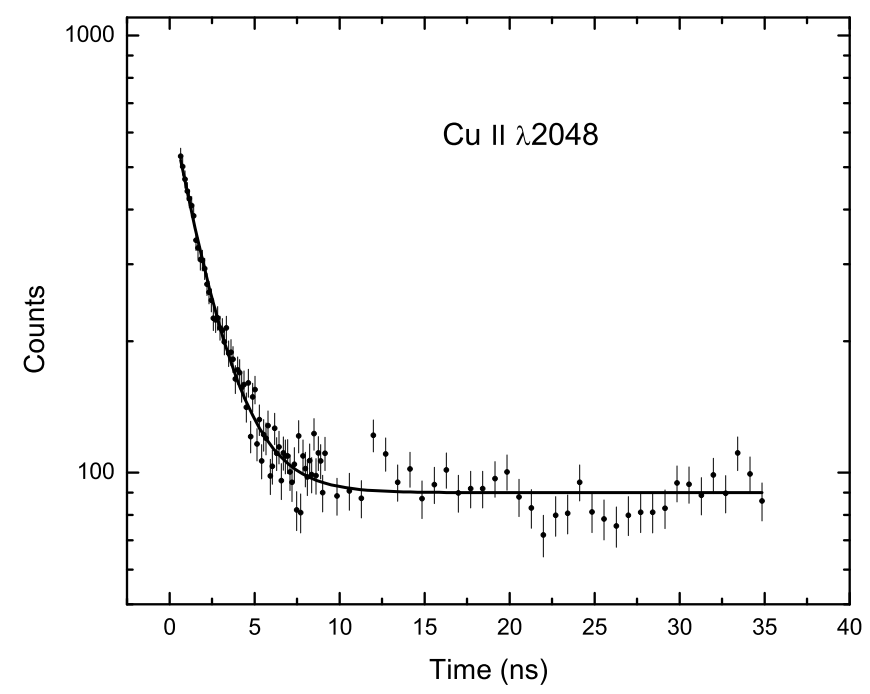

Figure 3-5: Semi-log plot with fit of forward decay curve at $170 \mathrm{keV}$ for $\lambda 2048$.

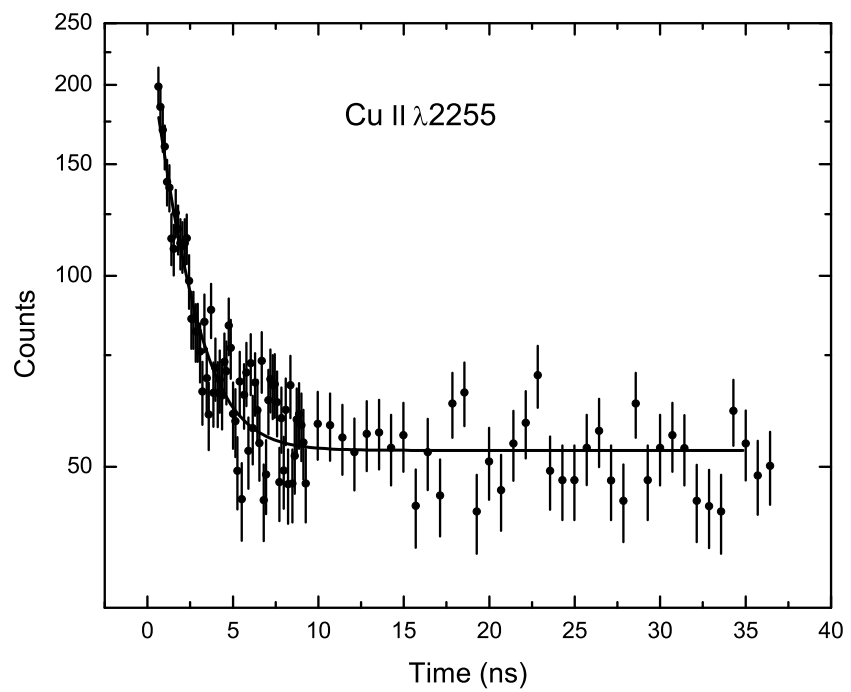

Figure 3-6: Semi-log plot with fit of forward decay curve at $170 \mathrm{keV}$ for $\lambda 2255$. 
The results were incorporated into an analysis to determine the importance of these transitions in repopulating the $4 \mathrm{p}^{1} \mathrm{P}_{1}^{o}$ level. The method of Arbitrarily Normalized Decay Curves (ANDC) of Curtis et al. (1971) was used. The ANDC analysis showed that $\lambda 2048$ played a role in repopulating the $4 \mathrm{p}^{1} \mathrm{P}_{1}^{\mathrm{o}}$ level, but not significantly enough to affect our results. 


\section{Chapter 4}

\section{Results and Discussion}

\subsection{Comparison of Lifetimes and Branching Ratios}

I compare the lifetimes measured using BFS with previously reported results. The lifetime for the $3 \mathrm{~d}^{9} 4 \mathrm{p}^{1} \mathrm{P}_{1}^{\mathrm{o}}$ level of $\mathrm{Cu}$ II is $1.37 \pm 0.04$ ns. This value was obtained by taking the weighted average of the three measurements made on $\lambda 1358$. The uncertainty was calculated by adding in quadrature the statistical uncertainties and the systematic uncertainty inferred from the spread in lifetimes under different experimental conditions.

When compared to the value reported from other experiments, this is in excellent agreement with the value of $1.34 \pm 0.22 \mathrm{~ns}$ reported by Pinnington et al. (1997) using fast beam/laser interaction. My value is shorter by slightly more than two standard deviations compared to the value of $1.8 \pm 0.2 \mathrm{~ns}$ reported by Curtis et al. (1976) using BFS and slightly more than one standard deviation from the measured value of 1.7 \pm 0.3 ns reported by Kono \& Hattori (1982) using delayed-coincidence techniques. 
Lifetimes were derived from a variety of theoretical methods. Theodosiou (1986) reported a lifetime of $1.056 \mathrm{~ns}$ using the Coulomb approximation (CA). Loginov (1993) obtained a lifetime of $0.940 \mathrm{~ns}$ from intermediate coupling (IC) calculations. Pinnington et al. (1997) reported a lifetime of 0.87 ns based on a relativistic Hartree-Fock (HFR) calculation. Donnelly et al. (1999) determined a value of 1.39 ns using configuration interaction (CI) plus relativistic effects. Biémont et al. (2000) obtained 0.84 ns using HFR plus core polarization. Finally, Dong \& Fritzsche (2005) reported a value of 1.15 ns with multi-configurational Dirac-Fock (MCDF) techniques. These results are summarized in Table 4.1 shown below. Both the length and velocity formalism was used by Donnelly et al. (1999) and Dong \& Fritzsche (2005); both results are listed, the length form first, in this table. Only the length results are shown for the branching fractions. The lifetimes of Donnelly et al. (1999) and Dong \& Fritzsche (2005) are most consistent with the two most recent experimental determinations, including the present one.

I also measured the lifetimes of two potential cascades into the upper state of interest, $\lambda \lambda 2048,2255$. The lifetime for the upper level of the $2048 \AA$ line was $1.88 \pm$ $0.06 \mathrm{~ns}$ and that of the $2255 \AA$ line was $1.65 \pm 0.13 \mathrm{~ns}$. Our values as well as several previously reported values are also summarized in Table 4.1; all results agree quite well. 
Table 4.1: Measured and Calculated Lifetimes and Branching Fractions of $\mathrm{Cu}$ II

\begin{tabular}{|l||c|c|c|c|}
\hline \multicolumn{1}{|c||}{} & \multicolumn{3}{c|}{ Lifetimes (ns) } & $\mathbf{B}_{u l} \lambda \mathbf{1 3 5 8}$ \\
\hline Upper Level & $4 \mathrm{p}^{1} \mathrm{P}_{1}^{\mathrm{o}}$ & $4 \mathrm{~d}^{1} \mathrm{~S}_{0}$ & $4 \mathrm{~d}^{3} \mathrm{D}_{1}$ & $\left(10^{8} \mathrm{~s}^{-1}\right)$ \\
\hline Wavelength $(\AA)$ & 1358 & 2048 & 2255 & \\
\hline Reference & & & & \\
\hline Experimental & & & & \\
This work & $1.37 \pm 0.04$ & $1.88 \pm 0.06$ & $1.65 \pm 0.13$ & 0.45 \\
Curtis et al. (1976) & $1.8 \pm 0.2$ & & & \\
Kono \& Hattori (1982) & $1.7 \pm 0.3$ & & & 0.255 \\
Pinnington et al. (1997) & $1.34 \pm 0.22$ & & & \\
Theoretical & & & & \\
Theodosiou (1986) & 1.056 & 1.498 & 1.319 & 0.484 \\
Loginov (1992) & 0.940 & & 1.53 & 0.468 \\
Pinnington et al. (1997) & 0.87 & & 1.72 & 0.576 \\
Donnelly et al. (1999) & $1.39 / 1.61$ & $1.72 / 1.79$ & $1.54 / 1.61$ & 0.440 \\
Biémont et al. (2000) & 0.84 & & 1.46 & 0.616 \\
Dong \& Fritzsche (2005) & $1.15 / 1.31$ & & $1.71 / 1.80$ & 0.46 \\
\hline
\end{tabular}

\subsection{Comparison of Oscillator Strengths}

I now compare my determination of the oscillator strength for the line of $\mathrm{Cu}$ II at $1358 \AA$ with those previously reported. To obtain an oscillator strength, or $f$-value,

I used

$$
f=\frac{g_{u}}{g_{l}}\left(\frac{\lambda}{2582.7}\right)^{2} A_{u l}
$$

where $f$ is the oscillator strength, $\lambda$ is the wavelength in $\AA$ and $\mathrm{A}_{u l}$ is the transition probability in $10^{9} \mathrm{~s}^{-1}$. The $g$-values are dependent on the $j$-values of the upper and lower levels of interest, respectively, and are given by $g=2 j+1$. For the $3 \mathrm{~d}^{10}{ }^{1} \mathrm{~S}_{0}$ $3 \mathrm{~d}^{9} 4 \mathrm{p}{ }^{1} \mathrm{P}_{1}^{\mathrm{o}}$ transition, $j_{u}$ is 1 and $j_{l}$ is 0 . The transition probability is related to the lifetime, $\tau$, in ns by $\mathrm{A}_{u l}=\mathrm{B}_{u l} \tau^{-1}$, where $\mathrm{B}_{u l}$ is the percentage of decays for the transition of interest relative to all decays from the upper level. Besides transitions 
to $3 \mathrm{~d}^{10}{ }^{1} \mathrm{~S}_{0}$, the ${ }^{1} \mathrm{P}_{1}^{\mathrm{o}}$ level decays to $3 \mathrm{~d}^{9} 4 \mathrm{~s}{ }^{3} \mathrm{D}_{2},{ }^{3} \mathrm{D}_{1}$, and ${ }^{1} \mathrm{D}_{2}$.

Using Equation 4.1, I obtain an $f$-value of $0.273 \pm 0.008$. This result is obtained with a branching fraction of 0.45 , the average of the theoretical results of Donnelly et al. (1999) and Dong \& Fritzsche (2005). As shown in Table 4.1, their lifetimes are most consistent with our precise determination, and their branching fraction is similar to the results of Theodosiou (1986) and Loginov (1993). The branching fractions of Pinnington et al. (1997) and Biémont et al. (2000) are substantially larger. Froese Fischer \& Glass (1980), who performed multi-configurational Hartree-Fock (MCHF) calculations, reported an $f$-value of 0.368 . Donnelly et al. (1999) gave 0.262 , and Biémont et al. (2000) obtained 0.602. Morton's compilation (2003) recommends an $f$-value of 0.263 for this transition, based mainly on the results of Donnelly et al. (1999). Table 4-2 shows a summary of the $f$-values. My oscillator strength agrees very well with that of Donnelly et al. (1999) which is essentially the same as Morton's (2003) recommended value of 0.263. Those of Froese Fischer \& Glass (1980) and Biémont et al. (2000) are significantly larger.

Table 4.2: Oscillator Strengths for the $3 \mathrm{~d}^{10}{ }^{1} \mathrm{~S}_{0}-3 \mathrm{~d}^{9} 4 \mathrm{p}{ }^{1} \mathrm{P}_{1}^{\mathrm{o}}$ transition of $\mathrm{Cu}$ II

\begin{tabular}{|l|c|}
\hline Reference & $f$-values \\
\hline This work & $0.273 \pm 0.008$ \\
Froese Fischer \& Glass (1980) & 0.368 \\
Donnelly et al. (1999) & 0.262 \\
Biémont et al. (2000) & 0.602 \\
\hline
\end{tabular}




\subsection{Concluding Remarks}

I have measured the lifetime of the $4 \mathrm{p}^{1} \mathrm{P}_{1}^{\mathrm{o}}$ state of $\mathrm{Cu}$ II. The value is in excellent agreement with the most recent experimental results and is in good agreement with the previous experimental results as well as with available theoretical lifetimes. While my oscillator strength is in very good agreement with that reported by Donnelly et al. (1999) and recommended by Morton (2003), those of Froese Fischer \& Glass (1980) and Biémont et al. (2000) are much larger.

The oscillator strength is used to determine the fraction of elements in the gases of the interstellar medium and, by extension, the fraction of those elements that are incorporated into dust grains. When trying to determine interstellar abundances, astronomers typically look to Morton's compilations for oscillator strength. Since my measured value for the oscillator strength is within $5 \%$ of the value recommended by Morton (2003), astronomers can be more confident about their conclusions. In particular, the $\mathrm{Cu}$ abundances described by Cartledge et al. (2006), which were based on the earlier recommendation of Morton (1991), need to be raised by a factor of 1.40. While the trends as a function of average gas density and path length noted by Cartledge et al. (2006) are not affected by this revision, it now appears there is less $\mathrm{Cu}$ incorporated into grains than once thought. 


\section{References}

Bevington, P. R., and Robinson, D. K. 1992, Data Reduction and Error Analysis for the Physical Sciences, (2 $2^{\text {nd }}$ ed.; McGraw-Hill College)

Biémont, E., Pinnington, E. H., Quinet, P., and Zeippen, C. J. 2000, Phys. Scripta, 61, 567

Cartledge, S. I. B., Lauroesch, J. T., Meyer, D. M., and Sofia, U. J. 2006, ApJ, 641, 347

Curtis, L. J., Berry, H. G., and Bromander, J. 1970, Phys. Scripta, 2, 216

Curtis, L. J., Engman, B., and Martinson, I. 1976, Phys. Scripta, 13, 109

Dong, C. Z., and Fritzsche, S. 2005, Phys. Rev. A, 72, 012507-1

Donnelly, D., Hibbert, A., and Bell, K. L. 1999, Phys. Scripta, 59, 32

Federman, S. R., Beideck, D. J., Schectman, R. M., and York, D. G. 1992, ApJ, 401,367

Froese Fischer, C., and Glass, R. 1980, Phys. Scripta, 21, 525 
Haar, R. R., Beideck, D. J., Curtis, L. J., Kvale, T. J., Sen, A., Schectman, R. M., and Stevens, H. W. 1993, NIMB, B79, 746

Jenkins, E. B., Savage, B. D., and Spitzer, Jr., L. 1986, ApJ, 301, 355

Kono, A., and Hattori, S. 1982, J. Opt. Soc. Am., 72, 601

Loginov, A. V. 1993, Phys. Scripta, 47, 38

Morton, D. C. 1991, ApJ Suppl, 77, 119

Morton, D. C. 2003, ApJ Suppl, 149, 205

Morton, D. C., and Smith, W. H. 1973, ApJ Suppl, 26, 333

Pinnington, E. H., Rieger, G., Kernahan, J. A., and Biémont, E. 1997, Can. J. Phys., 75, 1

Spitzter, Jr., L. 1978, Physical Processes in the Interstellar Medium, (John Wiley and Sons)

Theodosiou, C. E. 1986, J. Opt. Soc. Am. B, 3, 1107 